\title{
Real-time Queue Length Estimation Applying Shockwave Theory at Urban Signalized Intersections
}

\author{
Márton Tamás Horváth ${ }^{1 *}$, Tamás Tettamanti ${ }^{1}$ \\ ${ }^{1}$ Department of Control for Transportation and Vehicle Systems, Faculty of Transportation Engineering and Vehicle Engineering, \\ Budapest University of Technology and Economics, H-1111 Budapest, 3 Múegyetem rakpart, Hungary \\ * Corresponding author, e-mail: horvath.marton@mail.bme.hu
}

Received: 12 August 2020, Accepted: 30 June 2021, Published online: 26 July 2021

\begin{abstract}
Signal control is a basic need for urban traffic control; however, it is a very rough intervention in the free flow of traffic, which often results in queues in front of signal heads. The general goal is to reduce the delays caused, and to plan efficient traffic management on the network. For this, the exact knowledge of queue lengths on links is one of crucial importance. This article presents a linkbased methodology for real-time queue length estimation in urban signalized road networks. The model uses a Kalman Filter-based recursive method and estimates the length of the queue in every cycle. The input of the filter, i.e. the dynamics of queue length is described by the traffic shockwave theory and the store and forward model. The method requires one loop-detector per link placed at the appropriate position, for which the article also provides suggestions.
\end{abstract}

Keywords

urban traffic, queue length, shockwave, Kalman Filter

\section{Introduction}

Several measures of urban road traffic performance are in connection with the length of queues in the network, for example, total travel time, delay time, emission, and so on; therefore, queue length is considered as a fundamental measure [1].

Relevant data for estimation can be obtained from probe vehicles or fixed sensors, in most cases, loop-detectors, magnetic sensors, video cameras; or the combination of them [2]. Collected data can be processed in different ways; [3] divides them into stochastic and deterministic categories. Deterministic approaches use shockwave theorybased [4] models or analytical models based on the kinematic equations of vehicle movements. Stochastic learning approaches use models based on probability theory.

Sensors are fixed measurement points providing systematic data almost every signal cycle. Basic magnetic sensor-based techniques are presented in [5], tested on a motorway ramp. Number plate recognition data were exploited by [6], making it possible to estimate queue lengths for multiple lane sections as well. Moreover, this algorithm uses a Gaussian process interpolation model so that the license plate data set can be reconstructed even if some of it is missing.
Apart from vehicle identification at the upstream end and re-identification at the downstream end, even a single detector per lane per link placed at an appropriate position provides satisfactory results [2].

A critical aspect of traditional sensor-based data collection is that classical detectors can handle only queue lengths that are between the detector and the stop line. The extra queue length upstream to the detector is unknown, since the detector is supposed to be constantly occupied [7]. However, applying shockwave theory can handle this problem $[8,9]$. For the same purpose, [10] applied a two-layer approach by calculating link and network state simultaneously. The Lighthill-Whitham-Richards shockwave model [4], implemented in these algorithms, uses the principles of vehicle conservation and the fundamental diagram, which defines the connection between vehicle flow and density [11].

Cai et al. [12] combined the upstream detector data with probe vehicle trajectory data and identified critical points indicating queue growth and dissipation. A similar pattern recognition algorithm is applied in [13], where the essential pattern changes in intersection travel times and delays are identified. A major difference is that this algorithm uses probe vehicle data instead of fixed sensor data. 
Flexible, traffic responsive signal program data can also be an indicator of queue length. Liu et al. [7] combined this data with the shockwave theory and identified traffic state changes that distinguish queue discharge from upstream arrival traffic.

Probe vehicles can be even public transport buses if they are not separated from passenger cars in bus lanes [14]. Mathematical models often connect some theoretical fundamentals of traffic flow characteristics to probe vehicle data.

It should be noted that probe vehicle methods need relatively high market penetration [15], however, [16] states that even $2 \%$ of penetration can be enough. A large accuracy increase can be seen if penetration of observed vehicles reaches at least $10 \%$ [17]. Certainly, the actual penetration of observed vehicles is unknown. Zhao et al. [15] presented a method that first estimates the penetration of observed vehicles and then estimates queue length using probability theory.

Some other researchers estimated queue length and its deviation with Poisson vehicle arrival distribution [18], Markov chains [19], Bayesian Networks [17], or with deep learning algorithms [20].

The most similar method to our presented algorithm is elaborated by [21], applying Kalman Filter for predicting downstream arrivals. Two loop-detectors are used in each lane for determining the residual queue at the start of signal cycles. In contrast, our proposed method requires only one detector (closer to the upstream end) and applies the shockwave theory for the entire traffic flow instead of single vehicle identification.

The remainder of the paper is composed as follows: Section 2 describes the theoretical background of the techniques used for the model; Section 3 provides a detailed description of the model itself, Sections 4 and 5 show the simulation environment for model testing and the results. Concluding remarks are given in Section 6.

\section{Theoretical background}

This section introduces the two basic achievements that give the basis of the model: the Kalman Filter technique and the traffic shockwave theory.

\subsection{Kalman Filter}

The Kalman Filter, which is often used by traffic engineers, is a recursive method for linear filtering of discrete data [22]. Using this method, the state of a dynamic system can be precisely estimated, even if its exact nature is unknown.
The state of a sampled, discrete, time-invariant process is described by a linear difference equation:

$x(k+1)=A x(k)+B u(k)+v(k)$,

where $x(k) \in \mathbb{R}^{n}$ is the state vector, $u(k) \in \mathbb{R}^{m}$ is the optional control input vector, $v(k)$ is the process noise, $A \in \mathbb{R}^{n \times n}$ and $B \in \mathbb{R}^{n \times m}$ are coefficient matrices and $k=1,2, \ldots$ is the discrete timestep. The state vector is calculated in every $[k T,(k+1) T]$ time interval, where $T$ is the sampling time period.

The measurement equation of the system is:

$$
y(k)=C x(k)+w(k),
$$

where $y(k) \in \mathbb{R}^{p}$ represents measurement data, $w(k)$ is the process noise and $C \in \mathbb{R}^{p \times n}$ relates the measurement to the state. The Kalman Filter can be applied if process noise $v(k)$ and measurement noise $w(k)$ are assumed to be zero mean Gaussian white noise, which means that $E\{v(k)\}=0$ and $E\{w(k)\}=0$. The covariance matrices are assumed to be

$Q=E\left\{v(k) v(k)^{T}\right\}$,

$R=E\left\{w(k) w(k)^{T}\right\}$.

The Kalman Filter has a two-phase operation: prediction and correction. The prediction phase projects the current state and estimates ahead in time; the correction phase adjusts the previously projected estimate by the actual measurement. In the equations $\hat{x}^{-}(k)$ is the a priori state estimate at step $k$ and $\hat{x}(k)$ is the a posteriori state estimate at step $k$. The difference of estimated and actual values results the a priori and a posteriori estimate errors which determine the a priori and a posteriori estimate covariance $P^{-}(k)$ and $P(k)$. These represent the square of the estimated errors.

The prediction equations are the following:

$\hat{x}^{-}(k)=A \hat{x}(k-1)+B u(k-1)$,

projects the state ahead and

$P^{-}(k)=A P(k-1) A^{T}+Q$,

projects the error covariance ahead.

The correction equations are the following:

$K(k)=P^{-}(k) C^{T}\left(C P^{-}(k) C^{T}+R\right)^{-1}$,

computes Kalman gain $K$,

$\hat{x}(k)=\hat{x}^{-}(k)+K(k)\left(y(k)-C \hat{x}^{-}(k)\right)$, 
updates estimation with measurement $y(k)$ and

$$
P(k)=(I-K(k) C) P^{-}(k) \text {, }
$$

updates the error covariance [23].

\subsection{Urban shockwave theory}

Shockwaves are waves that originate from a sudden, substantial change in the state of the traffic flow. That is, a shock wave is defined by a discontinuity in the flow-density conditions in the time-space domain [24].

The basic principles of traffic shockwave theory for signalized urban road networks were first described by [25]. The model determines three basic traffic states: (i) the vehicles are in free flow and their velocity is chosen by the driver, (ii) the vehicles stand in the queue and their velocity is $v_{J}=0$, (iii) as the traffic light switches to green, vehicles leave the intersection moving by a $v_{C}$ critical velocity [26].

Two types of shockwaves are used in the traffic model presented in this article. The first one is the queuing shockwave velocity $\left(W_{1}\right)$ when moving vehicles stop as they reach the end of the queue standing at red light; the second one is the discharge shockwave velocity $\left(W_{2}\right)$ when vehicles standing in the queue start moving as the traffic light switches to green [27].

These shockwaves can be seen in Fig. 1, using a triangular fundamental diagram [28] based on [29].

\section{The queue length estimation model}

The estimation process is described by a linear discrete time-invariant state-space model. The numbers of vehicles in lanes on links, which are considered to be queue lengths, are estimated every time step according to the cycle time of traffic lights. Measurement is done by a single loop-detector per lane placed at the appropriate position.

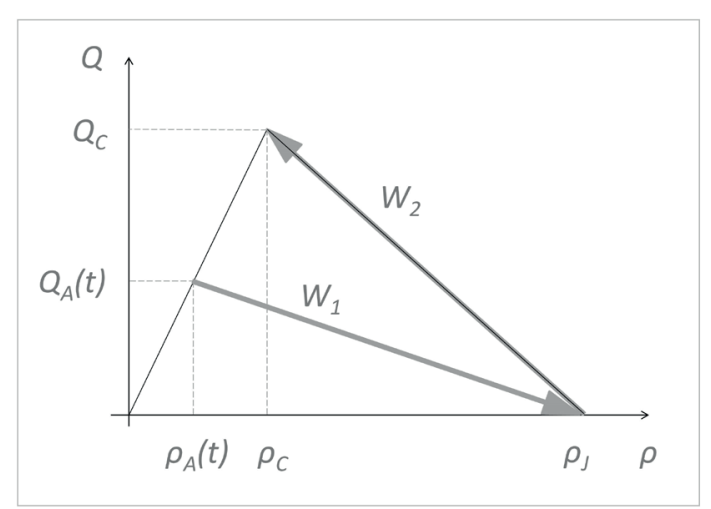

Fig. 1 Shockwaves on a triangular fundamental diagram. $W_{1}$ denotes the speed of queuing shockwave and $W_{2}$ denotes the speed of discharge shockwave. Q is traffic volume, $\rho$ is traffic density

\subsection{State equation and measurement equation}

The state equation describing the evolution of queue length is based on the store and forward model [30].

$x(k+1)=x(k)+q_{\text {in }}(k)-q_{\text {out }}(k)+v(k)$,

where $x(k)$ denotes the number of vehicles standing in the queue at the start of time step $k, q_{\text {in }}(k)$ and $q_{\text {out }}(k)$ represent the numbers of vehicles arriving and leaving the queue in time step $k$, and $v(k)$ is the noise of state dynamics.

The measurement equation (Eq. (14)) is obtained from the spatial occupancy of the link, i.e. the proportion of the link covered by cars.

$o_{s}(k)=\frac{x(k)}{L_{l} \cdot m \cdot \rho_{\text {jam }}}$,

where $o_{s}(k)$ is the spatial occupancy in time step $k, x(k)$ is the number of vehicles on the link in time step $k, L_{l}$ is link length, $m$ is the number of lanes, and $\rho_{j a m}$ is the density of vehicles during traffic jams, i.e. the maximum density occurring in real-life conditions.

The spatial occupancy itself cannot be effectively measured, but its value is considered to be similar to the time occupancy [31], the proportion of time in a cross-section during which any car was in it. Time occupancy in time step $k\left[o_{T}(k)\right]$ can be measured effectively. In this article a loop-detector is used for it but other sensor types can also be applied which are able to measure $o_{T}(k)$ in a cross-section. Spatial occupancy in time step $k\left[o_{s}(k)\right]$ can be obtained from time occupancy considering noise $z(k)$ as follows:

$o_{T}(k)=o_{s}(k)+z(k)$.

The measured number of vehicles $[y(k)]$ in time step $k$ is obtained as

$y(k)=L_{l} \cdot m \cdot \rho_{j a m} \cdot o_{T}(k)$.

Finally, the measurement equation is given as

$y(k)=x(k)+L_{l} \cdot m \cdot \rho_{j a m} \cdot z(k)$.

Noises $v(k)$ and $z(k)$ fulfill the stochastic assumptions of the Kalman Filter, i.e. they are independent, normal distribution white noise of which the expected values are 0 .

The proposed method is able to handle uncertainties within certain limits both in the state and the measurement equation, i.e., if the evolution of queue length is disturbed by non-observed vehicles (e.g., due to parking) or some detector measurements are not correct (e.g., a small vehicle is not sensed accurately). 


\subsection{Calculation of inflow and outflow based on shockwave theory}

Traffic inflow $q_{i n}(k)$ is determined by a shockwave model applied by [32].

$q_{\text {in }}(k)=t_{c y c} \cdot \rho_{j a m} \cdot W_{1}(k)$,

where $t_{c v c}$ is the cycle time of the traffic light, $\rho_{j a m}$ is the density of vehicles during traffic jams and $W_{1}(k)$ is the queuing shockwave velocity. $W_{1}(k)$ is formed by vehicles accumulating at red light, i.e., it is formed by vehicles joining the end of the queue:

$$
W_{1}(k)=\frac{q_{A}(k)}{\rho_{\text {jam }}-\frac{q_{A}(k)}{v_{\text {free }}}},
$$

where $q_{A}(k)$ is the number of vehicles measured by the sensor in time-step $k, \rho_{j a m}$ is the density of traffic during traffic jams, i.e., the maximum empirical density and $v_{\text {free }}\left(=v_{A}\right)$ is the velocity of vehicles while they are in free flow. In urban areas $v_{\text {free }}$ is around the speed limit. Variables $\rho_{\text {jam }}$ and $v_{\text {free }}$ are constant values that are calculated from reallife experience.

Traffic outflow $q_{\text {out }}(k)$ is also calculated by a shockwave model. When traffic light switches to green, the queue starts to discharge and the front of the queue moves backwards by $W_{2}$ speed. Parameter $W_{2}$ is assumed to be a constant value of $20 \mathrm{~km} / \mathrm{h}$ based on own real-life measurements in the city of Budapest and traffic simulations in Vissim microscopic traffic simulation software [33]. Both resulted in the above value. Traffic density of the queue is also known, as it is $\rho_{\text {jam }}$. The front of the queue discharges during the entire green time, however, not all vehicles that started moving can get through the traffic light, some will have to stop again. Therefore, not the entire green time can be taken into consideration when calculating the possible outflow. This part is defined as the effective time, when the outflow shockwave reaches the last vehicle that can exit the intersection during green time (see Fig. 2).

The outflow shockwave reaches this vehicle in $t_{\text {eff }}$ (effective time) and after that it starts moving and exits the link just at the end of green time. Certainly, if there are fewer vehicles in the queue, everyone can leave during green time. Calculation of the outflow requires no extra sensor; data of the inflow sensor can be used. First, the maximum expected outflow $q_{\text {out }}^{\max }$ is calculated:

$q_{\text {out }}^{\text {max }}=t_{\text {eff }} \cdot \rho_{\text {jam }} \cdot W_{2}$,

where $t_{\text {eff }}$ is the effective time, $\rho_{\text {jam }}$ is traffic density in the queue and $W_{2}$ is the speed of the discharge shockwave.

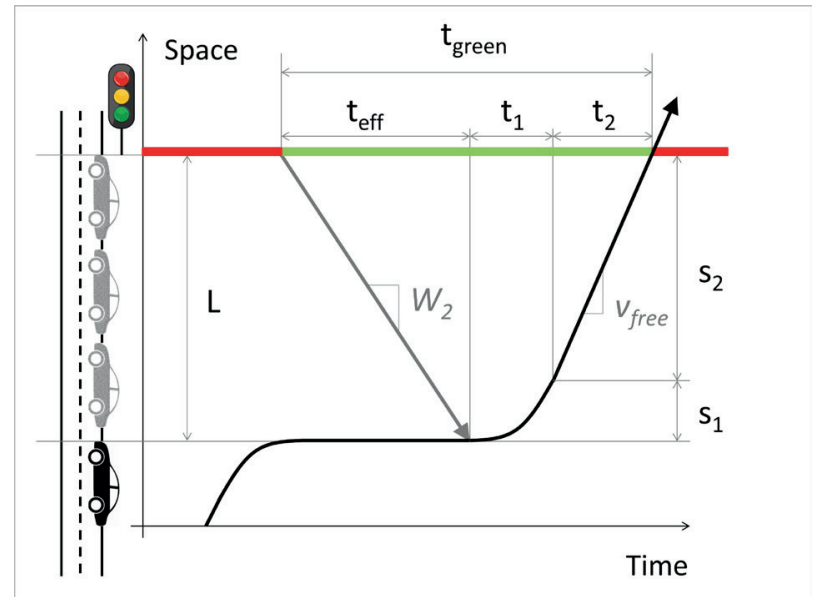

Fig. 2 Time-space diagram of the trajectory of the last vehicle that can exit the intersection. The vehicle can start moving in $t_{\text {eff }}$, after that it accelerates for $t_{1}$ until it reaches $v_{\text {free }}$, finally the vehicle moves with this constant speed for $t_{2}$, when it reaches the exit of the link

The calculation of effective time is based on the trajectory of the last vehicle that can exit the intersection, shown by Fig. 2. As the traffic light switches to green, the discharge shockwave starts to spread and reaches this last vehicle at $t_{\text {eff }}$ At this time, the vehicle is at position $L$.

$L=t_{\text {eff }} \cdot W_{2}$

The vehicle starts accelerating by $a$ for $t_{1}$ time until reaching its desired speed, which is considered to be $v_{\text {free }}$.

$v_{\text {free }}=t_{1} \cdot a$

Velocity can be a lower value than desired speed in oversaturated networks in gridlock situations. In these cases, a lower value can be used.

The vehicle covers $s_{1}$ distance during the acceleration phase.

$s_{1}=\frac{v_{\text {free }}^{2}}{2 a}$

When the vehicle reaches its desired speed $v_{\text {free }}$ then it moves on with constant speed and covers $s_{2}$ distance in $t_{2}$ time.

$s_{2}=t_{2} \cdot v_{\text {free }}$

Right at the end of green time, it leaves the intersection which means it has covered the same distance expressed in Eq. (18).

$L=s_{1}+s_{2}=\frac{v_{\text {free }}^{2}}{2 a}+t_{2} \cdot v_{\text {free }}$.

The effective time and the times of the accelerating and constant speed movement phases equal green time: 
$t_{\text {green }}=t_{\text {eff }}+t_{1}+t_{2}$.

Combining and arranging the results of Eq. (18) and Eq. (22), $t_{2}$ can be expressed as

$t_{2}=\frac{w_{2} \cdot t_{\text {eff }}}{v_{\text {free }}}-\frac{v_{\text {free }}}{2 a}$.

Finally, $t_{\text {eff }}$ can be obtained by substituting the results of Eq. (19) and Eq. (24) into Eq. (23):

$t_{\text {eff }}=\frac{v_{\text {free }} \cdot t_{\text {green }}-\frac{v_{\text {free }}^{2}}{2 a}}{v_{\text {free }}+W_{2}}$.

If green time is too short and the movement of the last exiting vehicle is continuously accelerating until exit, there is no constant speed phase, i.e., $t_{2}{ }^{*}=0$, which results in:

$t_{\text {green }}=t_{\text {eff }}^{*}+t_{1}^{*}$

and the vehicle does not achieve $v_{\text {free }}$ at the exit. (The borderline case is if the vehicle just reaches $v_{\text {free }}$.) Therefore, the position of the last exiting vehicle at the start of green time is calculated as

$L^{*}=\frac{a}{2} t_{1}^{* 2}$

where $L^{*}$ and $t_{1}^{*}$ denote the position of the last exiting vehicle and the time needed for it to exit the link, if it is not able to achieve its desired speed, because of too short green time.

The discharge shockwave has the same effect expressed in Eq. (18).

$$
L^{*}=t_{\text {eff }}^{*} \cdot W_{2} \text {, }
$$

where $t_{\text {teff }}^{*}$ denotes the effective time in the case when the vehicle is not able to achieve its desired speed, because of too short green time.

Combining Eqs. (26), (27), and (28) the value of $t_{1}^{*}$ can be expressed as follows:

$$
t_{1}^{*}=\frac{\sqrt{1+\frac{2 a}{W_{2}} \cdot t_{\text {green }}}-1}{\frac{a}{W_{2}}} .
$$

Note that mathematically $t_{1}^{*}$ could have two possible solutions, but the negative one is ignored.

Since $t_{2}{ }^{*}=0, t_{\text {teff }}^{*}$ can be obtained from Eq. (26) and Eq. (29) as follows: $t_{\text {eff }}^{*}=t_{\text {green }}-\frac{\sqrt{1+\frac{2 a}{W_{2}} \cdot t_{\text {green }}}-1}{\frac{a}{W_{2}}}$.

Finally, the borderline green time has to be calculated. Over this value Eq. (25) should be used to determine $t_{e f f}$, under it one should use Eq. (30). The borderline case is when the vehicle continuously accelerates and exits the link at the moment at which it has reached $v_{\text {free }}$. The borderline green time can be calculated from both previously introduced approaches by setting $t_{2}=0$ in Eq. (23) or $t_{1}^{*}=v_{\text {free }} / a$ in Eq. (27).

Considering that $v_{\text {free }}$ is $50 \mathrm{~km} / \mathrm{h}, a$ is $2 \mathrm{~m} / \mathrm{s}^{2}$ [34], and $W_{2}$ is $20 \mathrm{~km} / \mathrm{h}$, the borderline green time is $15.62 \mathrm{~s}$. Certainly, using different values for $v, a$ and $W_{2}$, this borderline will be different.

Using the previous results, the state equation can be rewritten. If there are fewer cars in the queue than $q_{\text {out }}^{\text {max }}$, it is assumed that all cars can exit the link during green time. Therefore, the state equation can be calculated as

$$
\begin{aligned}
& x(k+1)=x(k)+t_{c y c} \cdot \rho_{\text {jam }} \cdot \frac{q_{A}(k)}{\rho_{\text {jam }}-\frac{q_{A}(k)}{v_{\text {free }}}} \\
& -\min \left[t_{\text {eff }} \cdot \rho_{\text {jam }} \cdot W_{2} ; x(k)+t_{c y c} \cdot \rho_{\text {jam }} \cdot \frac{q_{A}(k)}{\rho_{\text {jam }}-\frac{q_{A}(k)}{v_{\text {free }}}}\right]+v(k) .
\end{aligned}
$$

\section{Simulation environment}

The performance of the model was investigated in Vissim microscopic traffic simulation network. The model is designed to be applied primarily on links of urban arterial corridors with signalized intersections where left turn movements (assuming right-side traffic) are separated from other movements in signal programs; i.e., these vehicles have their own green phase and own lanes and do not have to wait for the turning movement blocking the vehicles behind them. The test link with its inflows and outflows can be seen in Fig. 3. It should be noted that the exit at the perimeter is also ruled by a signal head so that residual traffic stays in the network at high traffic volumes.

Multiple simulations were run with different vehicle inputs, modeling increasing, decreasing, and stagnating queue lengths during different intensity of traffic volume. Measurement data of 6000 minutes were evaluated in 


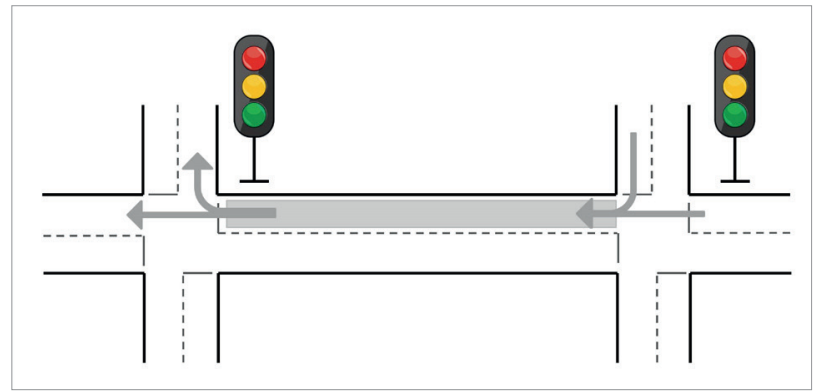

Fig. 3 Configuration of the test link and traffic directions

total, starting from an almost empty network until gridlock traffic jam situation. These measurement data were used to verify the state equation (Eq. (31)) and the measurement equation (based on Eq. (13)). Both the input and the output of these equations were measured in the simulation environment to obtain the exact state space representation and the measurement equation. Certainly, later, in real application, only the input values are used, and output values are calculated by the model.

The time step of calculation equals the cycle time, which was 60 seconds in this case.

The model presented in this article uses time occupancy as input measurement data. Since it can be measured at a fixed place, different occupancy values can be obtained at different locations of the link. Therefore, first, the optimal location of the detector was determined using simulation results. The connection between time-occupancy and queue length was observed.

The actual queue length, i.e. the output of the measurement equation was calculated in every time step applying the traditional store-and-forward model [30]. The occupancy, i.e. the input was measured on the whole length of the link by loop detectors placed at every 10 meters. After 6000 simulation runs these time occupancy measurement data were compared to the queue length in every period and the connection between the two datasets were calculated for every loop-detector. The aim of this process was to choose the detector on which the measured occupancy has the highest correlation with the actual queue length.

A linear connection is assumed between occupancy and queue length according to Eq. (13); therefore, linear regression was applied for loop-detector time-occupancy data and the queue length of the link. Regression was done for each detector. The highest correlation (a value of 0.9) was reached a bit upstream of the middle of the link and the lowest correlation (a value of 0.6) was experienced at the exit of the link. See Fig. 4 for detailed results. The darker the color is, the higher the correlation is. The test

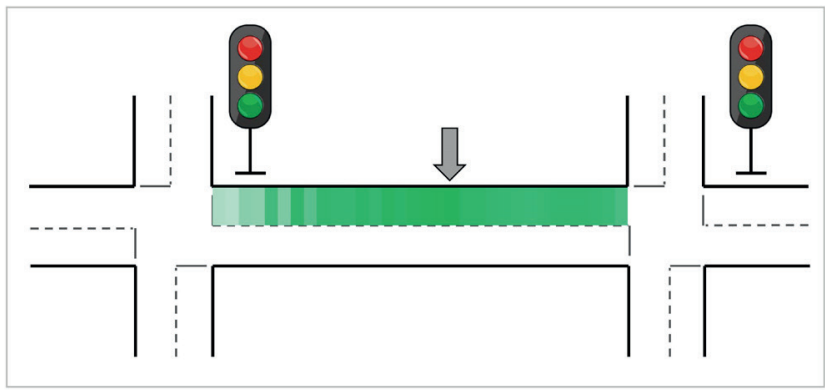

Fig. 4 Correlation between occupancy and queue length at different locations. The optimal location is marked with the arrow

link is $313 \mathrm{~m}$ long. The detector placed at the optimal location, marked by the grey arrow, was used for the model; this is the suggested location of the sensor.

It should be noted that 18 of the 31 measurement points resulted in higher correlation than 0.85 . These results show that the detector should be placed either at the middle of the link or a bit upstream of it or around the entrance.

\section{Simulation results}

The queue length estimation model was applied on the same network but with a different dataset in Vissim, and the results were evaluated in Matlab, using Kalman Filter for queue length estimation.

Vissim traffic input data was generated representing the daily traffic distribution of Budapest road traffic. Daily traffic flows were generated using the Budapest Macroscopic Transport Model [35]. Queue length was estimated on the test link (See Fig. 3) using this traffic input data.

Cycle time and hence the time step was 60 seconds. In this way 1440 measurement results were generated during simulation of the daily flow. The overall result can be seen in Fig. 5.

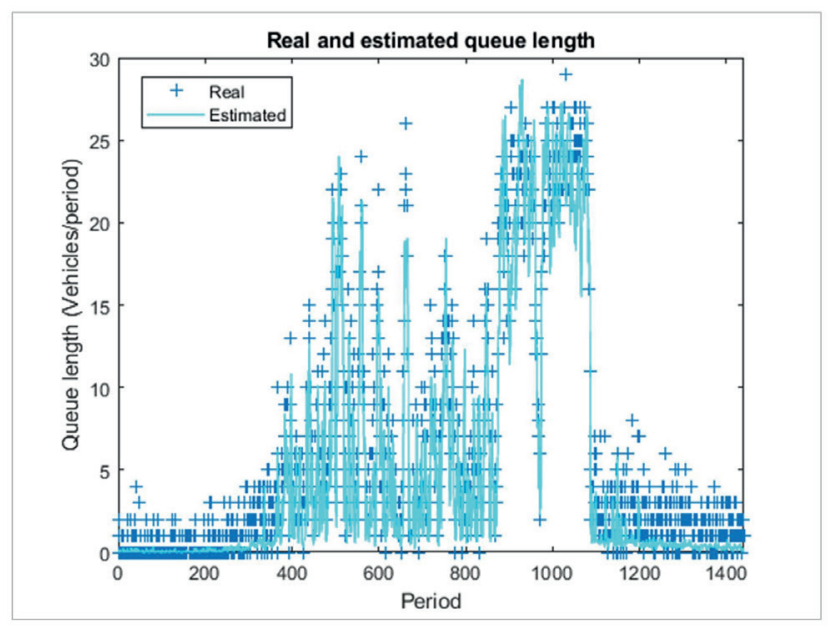

Fig. 5 Simulation results 
Fig. 6 shows the estimated queue length by Kalman Filter compared to the actual results calculated in Vissim. The gray line shows the theoretical optimum. When queue length is small, the filter slightly underestimates the actual state. Considering that low values mean short queue length, this does not matter too much; all vehicles queuing have the chance to get through the intersection in the next green phase. And this is what the filter states.

If there are many consecutive periods in which queue length is zero, the filter insists on this value even if there are a few single periods in which the queue length is above zero. The filter could be tuned to react more to these changes (i.e. not to underestimate queue length), but then the results would not be so accurate at higher queue lengths. For such cases, one can use time-varying filter parameters at lower and higher traffic volumes, like [36] did.

The performance of Kalman Filter is summarized in Table 1 by calculating the mean absolute error and root mean square error. The characteristics of the filter were evaluated separately for low and high traffic volumes and the overall performance was also taken into consideration.

The mean absolute error of 1440 measurements was 2.09 vehicles, which means that the filter miscalculates the actual queue length by 2.09 vehicles on average. Root mean square error is not much higher than mean absolute error, which means that there are not many large errors. Even though at low traffic volumes 2.09 is a relatively high difference (e.g., if 3 vehicles are predicted instead of 1

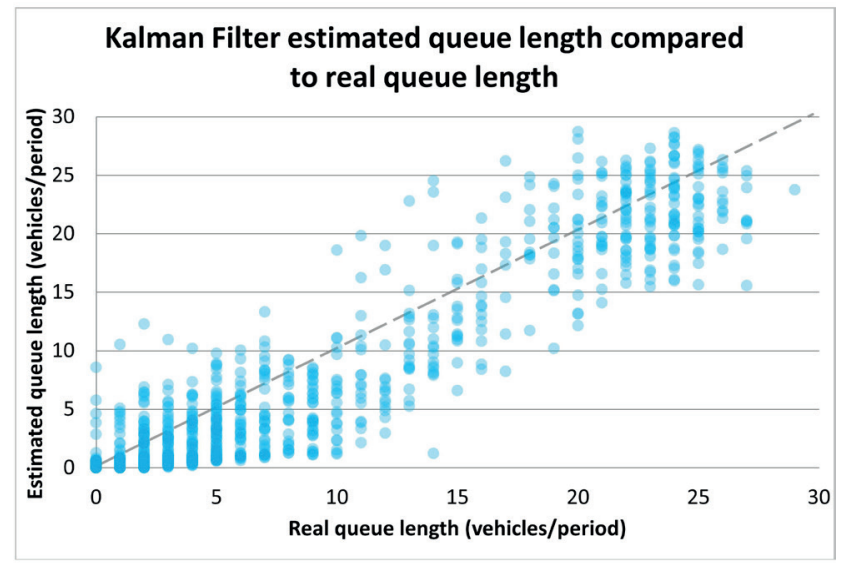

Fig. 6 Comparison of estimated and real queue lengths

Table 1 Performance of the filter (Low: Queue length < 15 vehicles; High: Queue length $\geq 15$ vehicles; Total: summarized data of Low and High)

\begin{tabular}{lccc}
\hline & $\begin{array}{c}\text { Low } \\
\text { volume } \\
\text { (vehicles) }\end{array}$ & $\begin{array}{c}\text { High } \\
\text { volume } \\
\text { (vehicles) }\end{array}$ & $\begin{array}{c}\text { Total } \\
\text { data } \\
\text { (vehicles) }\end{array}$ \\
\hline Mean Absolute Error & 1.85 & 3.24 & 2.09 \\
Root Mean Square Error & 2.64 & 3.96 & 2.91 \\
\hline
\end{tabular}

makes a difference of 200 percent), this is not significant in the sense that both real and estimated values are very low, this short queue will discharge in the next green phase. To be precise, the errors were also calculated separately for low (when real queue length is under 15 vehicles) and high (when real queue length is between 15 and 30 vehicles) volume traffic situations. The absolute error value was 1.85 at low volumes and 3.24 at high volumes. Relatively the latter value means circa $11-22 \%$ error on average (considering the cases when the queue length is 15 and 30 vehicles long). Root mean square errors are 0.8 higher than absolute mean errors in both cases; this is the same difference that can be seen during analysis of the whole dataset. This means there are not many significant errors in any of the two sub-datasets.

Analysis of the whole dataset containing daily traffic characteristics shows that the absolute difference of estimation is totally exact in $24 \%$ of cases. Another $24 \%$ result in a difference of 1 vehicle. Further $20 \%, 12 \%$, and $8 \%$ of cases result in a difference of 2, 3, and 4 vehicles. Errors exceed 4 vehicles in $12 \%$ of the cases (see Fig. 7).

The general conclusion is that estimating queue length using the method, an average error of 2 vehicles can be expected for low traffic volumes and an average error of 3 vehicles can be expected for high traffic volumes; $88 \%$ of cases result in a maximum error of 4 vehicles.

\section{Conclusions}

This paper introduced a shockwave theory-based real-time queue length estimation model using Kalman Filter. The method is designed to be used primarily on urban networks with signalized intersections.

The main contribution of the methodology is that it applies the effect of queuing and discharge shockwaves of traffic in a novel way by identifying the phenomenon of effective green time and calculating it based on kinematic equations using vehicle trajectories.

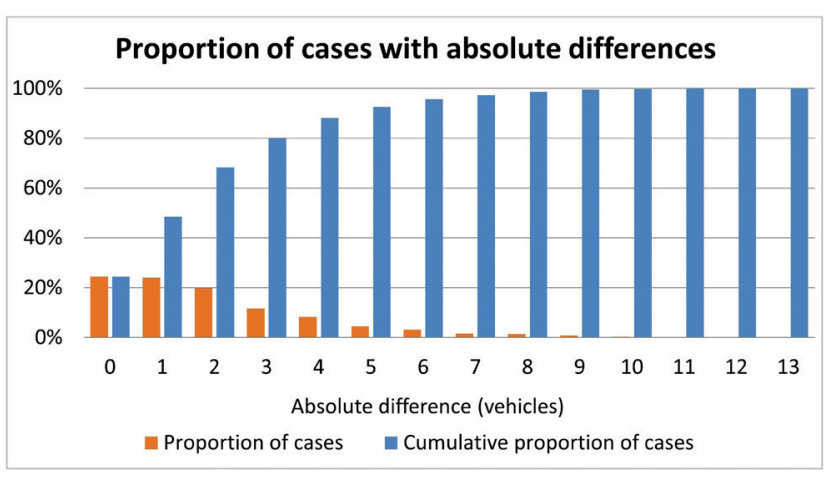

Fig. 7 Proportion of cases with absolute differences 
The methodology was tested in Vissim microscopic traffic simulation software and results show that the mean absolute error of queue length estimation is 2.09 vehicles on a $313 \mathrm{~m}$ long link. The absolute error of estimation does not exceed 4 vehicles in $88 \%$ of cases according to test results simulating daily traffic flow.

Further research focuses on treating the uncertainties of the model in a more precise way by testing a robust filtering model. The efficiency of the model can be increased by combining data from different sources; in our case, GPS data from probe cars [37] can be fused with detector data applying the Switching Kalman Filter [38]. Finally,

\section{References}

[1] Kisgyörgy, L., Rilett, L. R. "Travel Time Prediction by Advanced Neural Network", Periodica Polytechnica Civil Engineering, 46(1), pp. 15-32, 2002. [online] Available at: https://pp.bme.hu/ci/article/ view/617

[2] Li, H., Chen, N., Qin, L., Jia, L., Rong, J. "Queue length estimation at signalized intersections based on magnetic sensors by different layout strategies", Transportation Research Procedia, 25, pp. 16261644, 2017.

https://doi.org/10.1016/j.trpro.2017.05.212

[3] Guo, Q., Li, L., Ban, X. (J.) "Urban traffic signal control with connected and automated vehicles: A survey", Transportation Research Part C: Emerging Technologies, 101, pp. 313-334, 2019.

https://doi.org/10.1016/j.trc.2019.01.026

[4] Lighthill, M. J., Whitham, G. B. "On kinematic waves II. A theory of traffic flow on long crowded roads", Proceedings of the Royal Society A, Mathematical and Physical Sciences, 229(1178), pp. 317-345, 1955.

https://doi.org/10.1098/rspa.1955.0089

[5] Sanchez, R. O., Horowitz, R., Varaiya, P. "Analysis of Queue Estimation Methods Using Wireless Magnetic Sensors", Transportation Research Record, 2229(1), pp. 34-45, 2011. https://doi.org/10.3141/2229-05

[6] Zhan, X., Li, R., Ukkusuri, S. V. "Lane-based real-time queue length estimation using license plate recognition data", Transportation Research Part C: Emerging Technologies, 57, pp. 85-102, 2015. https://doi.org/10.1016/j.trc.2015.06.001

[7] Liu, H. X., Wu, X., Ma, W., Hu, H. "Real-time queue length estimation for congested signalized intersections", Transportation Research Part C: Emerging Technologies, 17(4), pp. 412-427, 2009. https://doi.org/10.1016/j.trc.2009.02.003

[8] Wu, A., Yang, X. "Real-time Queue Length Estimation of Signalized Intersections Based on RFID Data", Procedia - Social and Behavioral Sciences, 96, pp. 1477-1484, 2013. https://doi.org/10.1016/j.sbspro.2013.08.168

[9] Yao, J., Tang, K. "Cycle-based queue length estimation considering spillover conditions based on low-resolution point detector data", Transportation Research Part C: Emerging Technologies, 109, pp. 1-18, 2019.

https://doi.org/10.1016/j.trc.2019.10.003 the phenomenon of queue spillover to adjacent links can also be taken into consideration more directly in oversaturated networks.

\section{Acknowledgement}

The research reported in this paper was supported by the Higher Education Excellence Program in the frame of Artificial Intelligence research area of Budapest University of Technology and Economics (BME FIKP-MI/FM) as well as by Support for market-driven research, development and innovation projects (2019-1.1.1-PIACI-KFI-2019-00330).

[10] Rostami-Shahrbabaki, M., Safavi, A. A., Papageorgiou, M., Setoodeh, P., Papamichail, I. "State estimation in urban traffic networks: A two-layer approach", Transportation Research Part C: Emerging Technologies, 115, Article number: 102616, 2020. https://doi.org/10.1016/j.trc.2020.102616

[11] Cetin, M. "Estimating Queue Dynamics at Signalized Intersections from Probe Vehicle Data: Methodology Based on Kinematic Wave Model", Transportation Research Record, 2315(1), pp. 164-172, 2012. https://doi.org/10.3141/2315-17

[12] Cai, Q., Wang, Z., Zheng, L., Wu, B., Wang, Y. "Shock Wave Approach for Estimating Queue Length at Signalized Intersections by Fusing Data from Point and Mobile Sensors", Transportation Research Record, 2422(1), pp. 79-87, 2014.

https://doi.org/10.3141/2422-09

[13] Ban, X. (J.), Hao, P., Sun, Z. "Real time queue length estimation for signalized intersections using travel times from mobile sensors", Transportation Research Part C: Emerging Technologies, 19, pp. 1133-1156, 2011. https://doi.org/10.1016/j.trc.2011.01.002

[14] Kumar, S.V., Sivanandan, R. "Traffic Congestion Quantification for Urban Heterogeneous Traffic Using Public Transit Buses as Probes", Periodica Polytechnica Transportation Engineering, 47(4), pp. 257$267,2019$.

https://doi.org/10.3311/PPtr.9218

[15] Zhao, Y., Zheng, J., Wong, W., Wang, X., Meng, Y., Liu, H. X. "Various methods for queue length and traffic volume estimation using probe vehicle trajectories", Transportation Research Part C: Emerging Technologies, 107, pp. 70-91, 2019.

https://doi.org/10.1016/j.trc.2019.07.008

[16] Mei, Y., Gu, W., Chung, E. C. S., Li, F., Tang, K. "A Bayesian approach for estimating vehicle queue lengths at signalized intersections using probe vehicle data", Transportation Research Part C: Emerging Technologies, 109, pp. 233-249, 2019. https://doi.org/10.1016/j.trc.2019.10.006

[17] Wang, S., Huang, W., Lo, H. K. "Traffic parameters estimation for signalized intersections based on combined shockwave analysis and Bayesian Network", Transportation Research Part C: Emerging Technologies, 104, pp. 22-37, 2019. https://doi.org/10.1016/j.trc.2019.04.023 
[18] Comert, G., Cetin, M. "Analytical Evaluation of the Error in Queue Length Estimation at Traffic Signals From Probe Vehicle Data", IEEE Transactions on Intelligent Transportation Systems, 12(2), pp. 563-573, 2011. https://doi.org/10.1109/TITS.2011.2113375

[19] Geroliminis, N., Skabardonis, A. "Prediction of Arrival Profiles and Queue Lengths Along Signalized Arterials by Using a Markov Decision Process", Transportation Research Record, 1934(1), pp. 116-124, 2005 https://doi.org/10.1177/0361198105193400112

[20] Lee, S., Xie, K., Ngoduy, D., Keyvan-Ekbatani, M. "An advanced deep learning approach to real-time estimation of lane-based queue lengths at a signalized junction", Transportation Research Part C: Emerging Technologies, 109, pp. 117-136, 2019. https://doi.org/10.1016/j.trc.2019.10.011

[21] Lee, S., Wong, S. C., Li, Y. C. "Real-time estimation of lane-based queue lengths at isolated signalized junctions", Transportation Research Part C: Emerging Technologies, 56, pp. 1-17, 2015. https://doi.org/10.1016/j.trc.2015.03.019

[22] Welch, G., Bishop, G. "An Introduction to the Kalman Filter", University of North Carolina at Chapel Hill, Chapel Hill, NC, USA, Rep. TR95-041, 1995

[23] Tettamanti, T., Horváth, M. T., Varga, I. "Road Traffic Measurement and Related Data Fusion Methodology for Traffic Estimation", Transport and Telecommunication, 15(4), pp. 269-279, 2014. https://doi.org/10.2478/ttj-2014-0023

[24] Hoogendorn, S. P. "Traffic Flow Theory and Simultion, Chapter 08: Shock Wave analysis", University of Delft, Delft, Netherlands. [online] Available at: https://ocw.tudelft.nl/course-readings/chapter8-shock-wave-analysis/?course_id=8959 [Accessed: 2020/08/02]

[25] Hunt, P. B., Robertson, D. I., Bretherton, R. D., Winton, R. I. "SCOOT - A traffic responsive method of coordinating signals", Transport Research Laboratory, Wokingham, UK, Rep. 1014, 1981.

[26] Varga, B., Tettamanti, T. "Sztochasztikus lökéshullámmodell levezetése és alkalmazási lehetőségei" (Stochastic shockwave model and its possible applications), Közlekedéstudományi Szemle, 6(69), pp. 45-52, 2019. (in Hungarian) https://doi.org/10.24228/KTSZ.2019.6.4

[27] Wada, K., Ohata, T., Kobayashi, K., Kuwahara, M. "Traffic Measurements on Signalized Arterials from Vehicle Trajectories", Interdisciplinary Information Sciences, 21(1), pp. 77-85, 2015. https://doi.org/10.4036/iis.2015.77

[28] Seo, T., Kawasaki, Y., Kusakabe, T., Asakura, Y. "Fundamental diagram estimation by using trajectories of probe vehicles", Transportation Research Part B: Methodological, 122, pp. 40-56, 2019. https://doi.org/10.1016/j.trb.2019.02.005
[29] Newell, G. F. "A simplified theory of kinematic waves in highway traffic, Part I: General theory", Transportation Research Part B: Methodological, 27(4), pp. 281-287, 1993. https://doi.org/10.1016/0191-2615(93)90038-C

[30] Gazis, D. C., Potts, R. B. "The oversaturated intersection", In: Proceedings of the 2nd International Symposium on Traffic Theory, London, UK, 1963, pp. 221-237.

[31] Papageorgiou, M., Vigos, G. "Relating time-occupancy measurements to space-occupancy and link vehicle-count", Transportation Research Part C: Emerging Technologies, 16, pp. 1-17, 2008, https://doi.org/10.1016/j.trc.2007.06.001

[32] Varga, B., Tettamanti, T., Kulcsár, B., Qu, X. "Public transport trajectory planning with probabilistic guarantees", Transportation Research Part B: Methodological, 139, pp. 81-101, 2020. https://doi.org/10.1016/j.trb.2020.06.005

[33] PTV Group "PTV Vissim (Version 9)" [software] Available at: https://www.ptvgroup.com/en/solutions/products/ptv-vissim/ [Accessed: 2020/08/02]

[34] Bogdanovic, V., Ruskic, N., Papic, Z., Simeunovic, M. "The Research of Vehicle Acceleration at Signalized Intersection", Promet - Traffic and Transportation, 25(1), pp. 33-42, 2013. https://doi.org/10.7307/ptt.v25i1.1245

[35] Modell Tercett Consortium "Egységes Forgalmi Modell (EFM) Budapest és agglomerációjának teljes területére, Modellezési és használati útmutató", (Unified Transport Model for Budapest and its agglomeration, Transport Modeling Guidelines), BKK, Traffic Centre for Budapest, Budapest, Hungary, 2015.

[36] Sobolic, F. M., Bernstein, D. S. "Kalman-Filter-Based Time-Varying Parameter Estimation via Retrospective Optimization of the Process Noise Covariance", In: 2016 American Control Conference (ACC), Boston, MA, USA, 2016, pp. 4545-4550. https://doi.org/10.1109/ACC.2016.7526068

[37] Siroki, S., Nyerges, Á., Tihanyi, V. "A review of automated vehicle systems", In: 2019 International IEEE Conference and Workshop in Óbuda on Electrical and Power Engineering (CANDO-EPE), Budapest, Hungary, 2019, pp. 109-114. https://doi.org/10.1109/CANDO-EPE47959.2019.9111005

[38] Böker, G., Lunze, J. "Stability and performance of switching Kalman filters", International Journal of Control, 75(16-17), pp. 1269-1281, 2002. https://doi.org/10.1080/0020717021000023708 